\title{
The expanding constellation of immune checkpoints: a DNAMic control by CD155
}

\author{
Vincenzo Bronte \\ Department of Medicine, Verona University Hospital, Verona, Italy.
}

\begin{abstract}
The clinical benefits that have been achieved for a group of cancer patients with metastatic disease on checkpoint inhibitor therapy have kindled intense interest in understanding tumor-induced escape from T lymphocyte control. Other lymphoid cells also participate in tumor control; in particular, NK cells can limit hematogenous cancer metastasis spread and are also subject to negative regulation by developing cancers. In this issue of the $\mathrm{JCI}$, Li and colleagues define an unanticipated role for the stress-induced protein CD155 in cancer metastasis. The presence of CD155 on the surface of cancer cells was shown to promote tumor invasiveness, while its upregulation in tumor environment-infiltrating myeloid cells restrained antitumor immunity by impairing antitumor T lymphocytes and NK cell function. Together, these results support further exploration of strategies for targeting CD155.
\end{abstract}

Two-for-one: CD155 as a checkpoint and protumor gene The presence of CD155 in human cancers has been documented in some studies (1, 4) and is an unfavorable prognostic marker in cervical, urothelial, and lung cancer (5). $\mathrm{Li}$ and colleagues characterized the cellular distribution of CD155 protein in primary cutaneous melanomas by IHC. CD155 expression was prevalent on $\mathrm{HMB} 45^{+}$melanoma cells and various tumor-infiltrating myeloid cells, including $\mathrm{CD}^{-} 4^{+} \mathrm{CD} 11 \mathrm{c}^{-}$ macrophages, $\mathrm{CD} 14^{+} \mathrm{CD} 11 \mathrm{c}^{+}$myeloid cells, and $\mathrm{CD} 14^{-} \mathrm{CD} 11 \mathrm{c}^{+}$DCs, with negligible expression on tumor-infiltrating lymphocytes (TILs).

While CD155 expression had a variable and incomplete impact on the growth of different subcutaneously implanted tumors, lack of CD155 in mice strongly reduced the metastatic diffusion of tumors to the lungs. Moreover, CD155 had variable effects in different arms of the immune system, as primary tumor growth was mostly controlled by CD $8^{+} \mathrm{T}$ cells, but the absence of NK cells rendered both WT and $C d 155^{-/-}$mice unable to restrain lung metastasis formation (4).

Elegant bone marrow chimera experiments unveiled a distinct contribution of CD155 expression within the hematopoietic compartment in restraining tumor development (4). Specifically, ablation of CD155 in bone marrow-derived cells, both systemically in tumor-free mice and within the tumor microenvironment, enhanced DNAM-1 surface expression in circulating T lymphocytes and NK cells, as well as in TILs. Administration of an anti-DNAM-1-blocking $\mathrm{mAb}$ to $\mathrm{Cd} 155^{-/-}$mice abrogated $\mathrm{NK}$ cell-dependent control of metastatic spread, and, functionally, higher DNAM-1 expression was related to enhanced IFN- $\gamma$ cytokine production. These data suggest that CD155 acts in trans to inhibit DNAM-1 expression in different lymphoid cells, confirming and extending previously published data (6).

$\mathrm{Li}$ et al. also exploited the cancer cellautonomous effect of CD155 expression 
Primary tumor
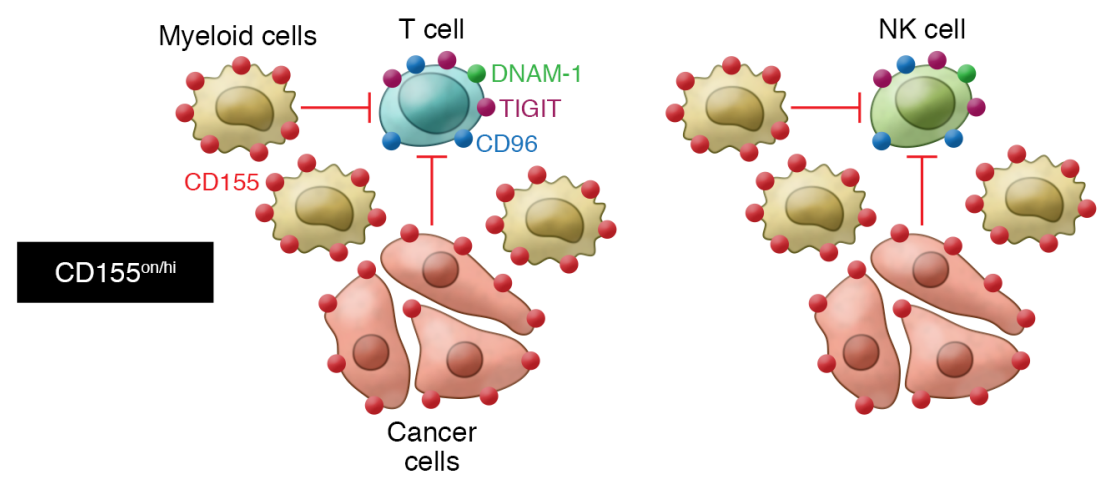

CD155 otho

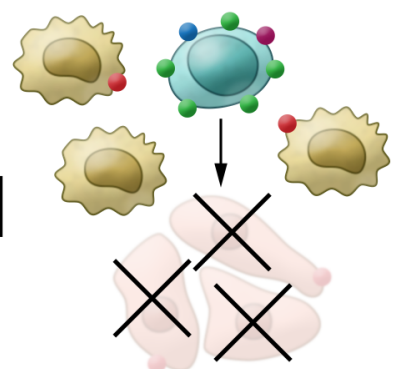

+ anti-PD-1

+ anti-TIGIT

+ anti-CD96
Metastatic niche

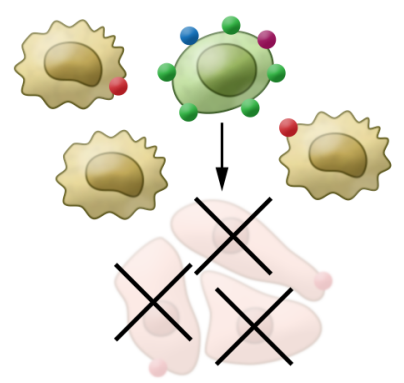

Figure 1. CD155 and CD155-mediated pathways have potential as therapeutic targets for limiting tumor growth. Within the cancer microenvironment of primary tumor lesions and the distant metastatic niche, the upregulation of CD155 (CD155 on/hi) enforces a decrease in the expression of DNAM-1 in both T lymphocytes and NK cells, impairing the antitumor activity exerted by these two immune effectors at these different sites. CD155 can also bind TIGIT and/or CD96 to induce inhibitory signals on NK cells. Genetic ablation (and possibly targeted inhibition by small molecules or antibodies; CD155 off/lo), combined with PD-1, TIGIT, and CD96 blockade, unleashes the full antitumor power at the primary tumor site. CD155 modulation as a single agent can have an effect on the control of metastases from NK cells.

and used the CRISPR-Cas9 system to delete CD155 in different, transplantable tumor cell lines, which were then inoculated in mice either intravenously or orthotopically (4). Although not all the cell lines were compared in these two settings, the results of these experiments provided a clear indication that tumor cells lacking CD155 have limited metastatic potential. CD155-deficient cancer grew more slowly in vivo and formed fewer metastases when inoculated in immunodeficient animals. In vitro, the number of cell colonies formed under limiting dilution and the ability of cells to migrate were both impaired by CD155 deficiency, whereas the rate of apoptosis under hypoxic or nutrient limitation conditions increased. Overall, these findings reveal a global inability of cancer cells to sustain autonomous survival when lacking CD155.

Given these experiments, it is obvious to surmise that manipulation of CD155 as a strategy to enhance immune-mediated rejection of tumors would probably require interference of both cancer and myeloid cell expression (Figure 1). Indeed, genetic ablation of CD155 in both host and cancer cells resulted in the greatest control of melanoma primary lesions and prevention of metastatic spread to the lungs (4). Moreover, anti-DNAM-1 mAb treatment ablated the beneficial anti-melanoma response achieved by CD155 loss only in Cd155deficient, but not WT, mice (4). These results indicate that, while DNAM- 1 is the main CD155-activating signal, DNAM-1independent mechanisms of the antitumor response operate in the lymphocytes of WT mice, and only the complete absence of CD155 in both tumor and immune compartments can unleash a preeminent DNAM-1 activation.

Finally, Li and colleagues focused on the therapeutic impact of CD155 modulation in combination with checkpoint inhibitor therapy (4). Genetic ablation of $C d 155$ in mice enhanced the response to anti-PD-1 mAb and to CTLA4/PD-1 blockade against various subcutaneously growing tumors. It is worth noting that triple blockade of TIGIT, CD96, and PD-1 had a higher therapeutic index in WT mice bearing a CD155-deficient melanoma than in animals that received control tumor cells. As both TIGIT and CD96 bind CD155 and induce negative signals that restrain DNAM-1-dependent activation, the inability of TIGIT and CD96 blockade to restrain the negative influence of CD155 on the surface of cancer cells confirms the hypothesis that CD155 can directly modulate DNAM-1, probably by reducing its expression on TILs.

\section{Open questions and future directions}

The manuscript by $\mathrm{Li}$ and colleagues extends our knowledge of the intricate interplay between immune-activating molecules and their control circuits, an aspect that provides multiple avenues to ensure appropriate responses. DNAM-1 and other NK cell-activating receptors are indeed under the control of additional checkpoints that operate under steady-state conditions. For example, IL-1 receptor 8 (IL-1R8) is a negative regulator of NK cell maturation and effector function, as genetic Il1r8 ablation leads to a cell-autonomous, IL-18-dependent increase in mature NK cell numbers and enhances IFN- $\gamma$ production upon ex vivo stimulation (7). Compared with WT animals, Ill $18^{-/-}$mice are more resistant to solid tumors in organs in which NK cells are more frequent, such as the liver and 
lungs, are less prone to developing lung and liver metastases from sarcoma and colon carcinoma lines, respectively, and have a lower incidence of hepatocellular carcinoma after chemical-induced carcinogenesis (7). From a cancer immunology viewpoint, a lack of CD155 and IL-1R8 produces overlapping outcomes. However, it must be pointed out that IL-1R8 seems to be restricted to NK cells. The obvious question being, is there any benefit to targeting these different checkpoints at the same time?

Even though additional studies on different cancer types are necessary, the pattern of CD155 distribution within the tumor environment matches PD-L1 expression, with both cancer and myeloid cells primarily expressing the two proteins. This thought-provoking parallelism opens up a few issues that will require further analyses. The signals driving CD155 upregulation in either cancer cells or myeloid cells are not fully understood and might be dissimilar, with some evidence pointing to oncogenes, DNA damage, and the stress response as mediators of CD155 expression (8). In the case of PD-L1 regulation, a clear contribution of IFN- $\gamma$ released from activated immune cells has been documented (9), and host cells expressing PD-L1 might be more important than tumor cells in driving $\mathrm{T}$ lymphocyte exhaustion and the response to PD-1/ PD-L1 blockade in experimental and human cancers, as was recently reported $(10,11)$. It remains to be determined whether IFN- $\gamma$ also controls CD155. Moreover, considering the evidence provided by Li et al., it will be important to correlate the presence of CD155 in human cancers with DNAM-1 downregulation in both circulating and tumor-infiltrating $\mathrm{T}$ lymphocytes and NK cells.

The cancer cell-intrinsic activity of CD155 also demands deeper exploration of the molecular pathways involved and the potential involvement of CD155 in the transformed phenotype. While a contribution of CD155 to tumor cell invasion and migration through the interplay with integrins and focal adhesion kinase is supported by in vitro experimental data $(12,13)$, the molecular links to epithelial-to-mesenchymal transition, cell cycle, and cell survival need to be explored.

The clinical translation of these results and the exploitation of ideal immunotherapy combinations will rely on the necessary step of developing strategies to target and block CD155. Both small molecules and antibodies can be designed for this purpose. Indeed, $\mathrm{Li}$ and colleagues show that anti-CD155 mAb mimics genetic Cd155 ablation and increases DNAM-1 in both $\mathrm{T}$ lymphocytes and NK cells of tumor-free mice (4). Additional data about the antitumor efficacy of this $\mathrm{mAb}$, when administered alone or in combination with other checkpoint inhibitors, will be needed to pave the way for further development of this strategy. It must be stressed that any new CD155-targeting drugs will have to balance the DNAM-1/ CD155 and TIGIT/CD96/CD155 axes to optimize the full activation of anticancer immune effectors. In theory, the best approach should maximize the blockade of CD155 interactions with TIGIT and CD96 and disrupt the negative influence of CD155 on DNAM-1 expression, but allow DNAM-1 to recognize CD155 and drive a full activation signal.

One final but important aspect of evaluating the effect of CD155 targeting for cancer therapy concerns the choice of tumor models used to assess combination therapy experiments, on the basis of the control of transplanted primary tumor growth. As exemplified by the findings of Li et al. about the split antitumor immune response, it is likely that these experiments address the key impact on the exhausted $\mathrm{CD}^{+} \mathrm{T}$ cell compartment. It will be essential to establish whether combination therapy also affects the NK cell-mediated control of metastatic disease, further improving the already remarkable effect of CD155 modulation.

\section{Acknowledgments}

This work was supported by grants from the Italian Association for Cancer Research (AIRC) (IG grants 6599, 12182, and 14103 and the Special Program for Molecular Clinical Oncology 5 per mille) and by a grant from the Italian Ministry of Health (FINALIZZATA RF-201102348435 cup:E35G1400019001).

Address correspondence to: Vincenzo Bronte, Immunology Section, Department of Medicine, P.Le L.A. Scuro 10, 37124, Verona. Phone: 39.045.8124007; Email: vincenzo.bronte@univr.it.

1. López-Soto A, Gonzalez S, Smyth MJ, Galluzzi L. Control of metastasis by NK cells. Cancer Cell. 2017;32(2):135-154.

2. Chan CJ, et al. The receptors CD96 and CD226 oppose each other in the regulation of natural killer cell functions. Nat Immunol. 2014;15(5):431-438.

3. Subrahmanyam PB, et al. Distinct predictive biomarker candidates for response to anti-CTLA-4 and anti-PD-1 immunotherapy in melanoma patients. JImmunother Cancer. 2018;6(1):18.

4. Li X, et al. CD155 loss enhances tumor suppression via combined host and tumorintrinsic mechanisms. JClin Invest. 2018;128(6):2613-2625.

5. PVR Pathology. The Human Protein Atlas. PVR Website. https://www.proteinatlas. org/ENSG00000073008-PVR/pathology. Accessed April 19, 2018.

6. Seth S, et al. Intranodal interaction with dendritic cells dynamically regulates surface expression of the co-stimulatory receptor CD226 protein on murine T cells. J Biol Chem. 2011;286(45):39153-39163.

7. Molgora M, et al. IL-1R8 is a checkpoint in NK cells regulating anti-tumour and anti-viral activity. Nature. 2017;551(7678):110-114.

8. Cerboni C, et al. The DNA damage response: a common pathway in the regulation of NKG2D and DNAM-1 ligand expression in normal, infected, and cancer cells. Front Immunol. 2014;4:508.

9. Spranger S, et al. Up-regulation of PD-L1, IDO, and $\mathrm{T}$ (regs) in the melanoma tumor microenvironment is driven by CD8(+) T cells. Sci Transl Med. 2013;5(200):200ra116.

10. Tang H, et al. PD-L1 on host cells is essential for PD-L1 blockade-mediated tumor regression. J Clin Invest. 2018;128(2):580-588.

11. Lin $\mathrm{H}$, et al. Host expression of PD-L1 determines efficacy of PD-L1 pathway blockademediated tumor regression. JClin Invest. 2018;128(4):1708.

12. Sloan KE, et al. CD155/PVR plays a key role in cell motility during tumor cell invasion and migration. BMC Cancer. 2004;4:73.

13. Sloan KE, Stewart JK, Treloar AF, Matthews RT, Jay DG. CD155/PVR enhances glioma cell dispersal by regulating adhesion signaling and focal adhesion dynamics. Cancer Res. 2005;65(23):10930-10937. 